\title{
Absolute volume of the rectum and AUC from rectal DVH between 25Gy and 50Gy predict acute gastrointestinal toxicity with IG-IMRT in prostate cancer
}

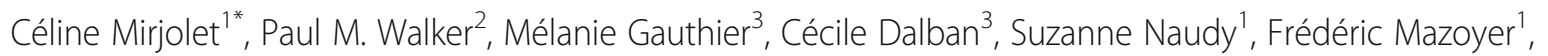
Etienne Martin ${ }^{1}$, Philippe Maingon ${ }^{1}$ and Gilles Créhange ${ }^{1,2}$

\begin{abstract}
Background: To determine whether dose/volume specific endpoints (DVSE) or Area under the rectal DVH curve ( $\mathrm{A} A \cup C$ ) better predict acute gastrointestinal (GI) toxicity in prostate cancer patients treated with IMRT in the era of daily image guidance (IG-IMRT).

Methods: A set of DVSE was recorded from V25 to V75 (increments of 5Gy) (both in \% and in cc) for 180 men. The rAUC was calculated for doses ranging between 25Gy and 50Gy ( AUUC $_{25-50}$ ). Univariate and multivariate logistic regressions were performed to determine the relationship between DVSE or $\mathrm{AUUC}_{25-50}$ and the appearance of any acute Gl toxicity.

Results: The rates of acute grade 1 (G1), G2 and G3 Gl toxicities were $53.3 \%, 10.6 \%$ and $1.1 \%$, respectively. No G4 + toxicity was observed.

Rectal V25 to V75 expressed in \% were not predictive of $G \geq 1$ GI toxicity ( $p \geq 0.12)$ whereas rectal V25 to V50 expressed in $c c$ did correlate with $G$ toxicity $G \geq 1$ ( $p \leq 0.04$ ). rAUC $25-50$ expressed in $c c$. Gy correlated significantly with the occurrence of any acute $\mathrm{Gl}$ toxicity $\mathrm{G} \geq 1(p=0.027)$.
\end{abstract}

Conclusions: The absolute volume of the rectum between 25Gy and 50Gy and $\mathrm{rAUC}_{25-50}$ could significantly predict any acute rectal toxicity in prostate cancer patients treated with daily IG-IMRT.

Keywords: Prostate cancer, Acute rectal toxicity predictive factor, Radiotherapy

\section{Background}

Intensity Modulated Radiation Therapy (IMRT) with daily image guidance of soft tissues in patients with localized prostate cancer $(\mathrm{PCa})$ has been shown to improve biochemical control and to reduce rectal toxicities compared with 3-dimensional conformal radiation therapy (3DCRT) [1-3]. The risk of normal tissue complications is typically evaluated from the amount of tissue exposed to a given dose (namely, dose-volume specific endpoint (DVSE)). For 3D-CRT, DVSE have been demonstrated to be reliable and reproducible for predicting acute and late

\footnotetext{
* Correspondence: cmirjolet@cgfl.fr

'Department of Radiation Oncology, Centre Georges Francois Leclerc, 1, rue du Pr Marion, 21049 Dijon, France

Full list of author information is available at the end of the article
}

toxicity according to Radiation Therapy Oncology Group (RTOG) scoring criteria [4]. Several dose-constraint guidelines, based on post-hoc analyses of rectal toxicity and its correlation with each DVSE, have been established to determine what relative volume of rectum (in \%) can safely receive high doses [5-8].

As for image-guided IMRT (IG-IMRT), there are no robust, reproducible data in the literature to indicate which DVSE are useful for a more accurate prediction of acute toxicity. Guidelines used by radiation clinicians were based on late toxicities and came mostly from 3D conformal radiotherapy [4]. Regarding the lower rates of rectal toxicity observed with IG-IMRT compared with 3D-CRT [9], standard DVSE developed from 3D-CRT results could be irrelevant. Hence, new tools are needed 
for IG-IMRT to improve predictions for any grade of acute toxicity. The purpose of this study was to determine a new type of dose/volume parameter to predict any acute GI toxicity with daily IG-IMRT. This new parameter was determined by studying a set of several DVSE expressed in \% and in cc. As the shape of the entire rectal DVH curve from low to high doses cannot be reflected by one single DVSE or even several DVSE, we also investigated the area under the rectum DVH curve (rAUC) as a challenger for predicting acute GI toxicity.

\section{Methods}

\section{Selection of patients}

We selected 180 men with at least eight visits for toxicity evaluations and with available DVH data. All of the men had localized PCa treated with daily IG-IMRT with curative intent.

Characteristics of patients are summarized in Table 1.

\section{IMRT}

All patients first underwent a planning CT scan with $2.5 \mathrm{~mm}$ slice thickness in the supine position with knee and ankle supports. A rectal enema was given before the $\mathrm{CT}$ for each patient. They were asked to maintain the same degree of bladder filling during the simulation and treatment sessions. Critical normal-tissue structures were outlined by a radiation oncologist on each axial CT image. The rectum was defined as a cylindrical structure around the outer rectal wall and contoured from the ischial tuberosities to the rectosigmoid junction, identified in accordance with international guidelines by the level at which the GI tract narrows and diverges anteriorly from the rectum $[4,10]$.

Intensity was modulated by dynamic multileaf collimation using the sliding window technique, as previously described by our group in this journal $[10,11]$. Patients who underwent whole pelvic radiotherapy were excluded from this study. The median dose prescribed to the prostate PTV was 78Gy [74-80] at 2Gy per fraction and five fractions per week.

\section{IGRT}

Daily on-line repositioning based on soft-tissues was performed for all the patients using either $\mathrm{kV}$ Cone Beam Computed Tomography or a 3D ultrasound system as described in detail elsewhere [11, 12].

\section{Dose/volume modeling for rectal toxicity}

A set of standard DVSEs was tested: the volume of the rectum receiving from 25Gy to 75Gy (V25 to V75), expressed in percentages (\%) and in cubic centimeters (cc).
Table 1 Characteristics of patients and treatments

\begin{tabular}{|c|c|}
\hline & All patients \\
\hline & $N=180$ \\
\hline Age (median [range]) years & 70.5 [49.6-84.9] \\
\hline \multicolumn{2}{|l|}{ T stage-n (\%) } \\
\hline T1c & 57 (31.7\%) \\
\hline T2a & $31(17.2 \%)$ \\
\hline $\mathrm{T} 2 \mathrm{~b}$ & $37(20.6 \%)$ \\
\hline $\mathrm{T} 2 \mathrm{C}$ & 19 (10.6\%) \\
\hline T3а & $30(16.7 \%)$ \\
\hline $\mathrm{T} 3 \mathrm{~b}$ & $6(3.3 \%)$ \\
\hline PSA (ng/ml) (median [range]) & 10 [0.8-99] \\
\hline \multicolumn{2}{|l|}{ Gleason score-n (\%) } \\
\hline$\leq 6$ & $103(57.2 \%)$ \\
\hline 7 & $64(35.6 \%)$ \\
\hline$\geq 8$ & $13(7.2 \%)$ \\
\hline \multicolumn{2}{|l|}{ Risk groups-n (\%) } \\
\hline Low risk & $48(26.7 \%)$ \\
\hline Intermediate risk & 68 (37.8 \%) \\
\hline High risk & $64(35.6 \%)$ \\
\hline \multicolumn{2}{|l|}{ Hormone therapy-n (\%) } \\
\hline Neoadjuvant & $50(27.8 \%)$ \\
\hline Concomitant & 69 (38.3\%) \\
\hline Adjuvant & 70 (38.9\%) \\
\hline Radiotherapy: prostate dose $\left(2 \mathrm{~Gy} / \mathrm{fx}^{\mathrm{a}}\right)$ & $78[70-80]$ \\
\hline \multicolumn{2}{|l|}{ Median [range] } \\
\hline \multicolumn{2}{|l|}{ TURP $^{\mathrm{b}}-\mathrm{n}(\%)$} \\
\hline Yes & 35 (19.7\%) \\
\hline No & $143(80.3 \%)$ \\
\hline Missing & $2(1.1 \%)$ \\
\hline
\end{tabular}

Calculation of the area under the rectum DVH curve (rAUC) In the second step, we calculated the area under the DVH curve between 25 and 50Gy for the rectum $\left(\right.$ rAUC $\left._{25-50}\right)$.

The respective rAUC, expressed in cc.Gy, were calculated every 5Gy in the following manner (Fig. 1):

$$
\mathrm{rAUC}_{\mathrm{doseX}-\mathrm{X}+5 \mathrm{~Gy}=5} * \mathrm{~V}_{\mathrm{X}+5 \mathrm{~Gy}}+2.5 *\left(\mathrm{~V}_{\mathrm{XGy}}-\mathrm{V}_{\mathrm{X}+5 \mathrm{~Gy}}\right)
$$

For example (Fig. 1): $\operatorname{rAUC}_{25-30}=5 * \mathrm{~V} 30+2.5 *$ (V25 - V30)

Thus, $\mathrm{rAUC}_{25-50}=\mathrm{rAUC}_{25-30}+\mathrm{rAUC}_{30-35}+\mathrm{rAUC}_{35-40}$ + rAUC $_{40-45}+$ rAUC $_{45-50}$

\section{Follow-up and toxicity evaluation}

Each patient was seen in our institution every week during the radiation therapy and at 3 months and 6 months 


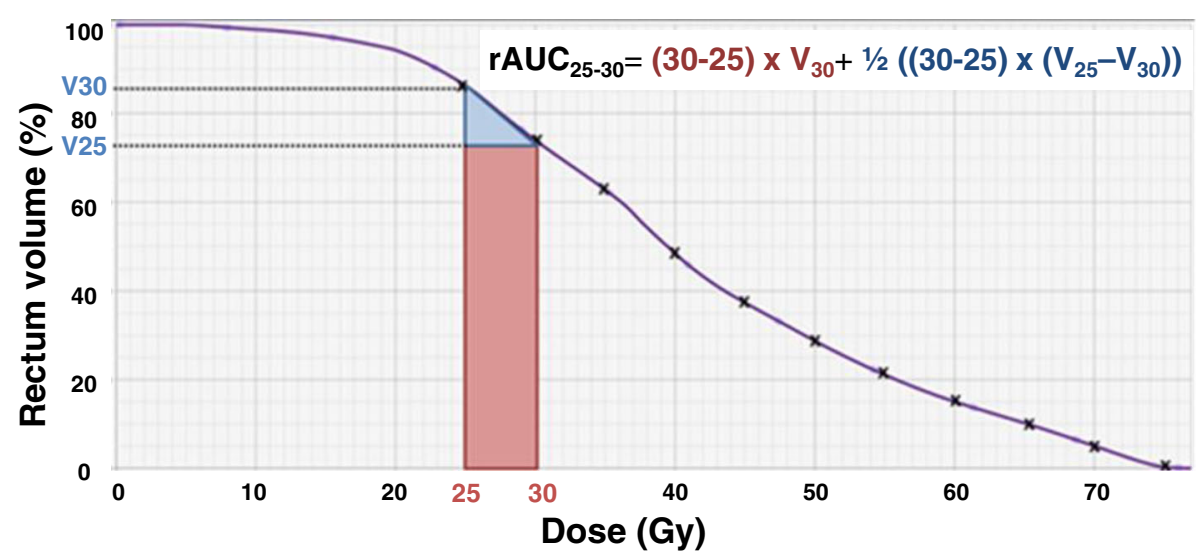

Fig. 1 Illustration of rAUC 5Gy calculation from 25 to 30Gy (rAUC 25-30). Legends: DVH: Dose Volume Histogram, V25 = volume of rectum receiving $25 \mathrm{~Gy} ; \mathrm{V} 30=$ volume of rectum receiving 30Gy; $\mathrm{AUUC}_{25-30}=$ Area Under the Curve of rectal DHV between 25 and $30 \mathrm{~Gy}$

thereafter. Acute toxicity was evaluated and scored using the Common Toxicity Criteria Adverse Events scales (CTCAE) version 3.0 weekly during each week of radiotherapy and 3 months after the completion of the radiotherapy. As acute toxicities can last for 3 months, we chose to extend our evaluation to 6 months. The worse GI toxicity (diarrhea, constipation, hemorrhoids, rectal hemorrhage, anal incontinence, proctitis and anitis) grade of each patient was analyzed.

\section{Statistical analyses}

Mann-Whitney tests were used to determine the relationship between V25-V75, expressed in \% and in cc or rAUC $_{25-50}$ and the appearance of any acute GI toxicity $(\mathrm{G} \geq 1)$.

The optimal AAUC $_{25-50}$ cut-off value related to acute GI toxicity was determined using a ROC curve with Youden's index. Univariate and multivariate logistic regressions were used to describe the acute GI toxicity $(\mathrm{G} \geq 1)$ by estimating the Odds-Ratio and $95 \%$ confidence interval (CI). The multivariate model included the optimal rAUC $_{25-50}$ cut-off value and the adjustment parameters (age, transurethral resection of the prostate (TURP), hormone therapy and rectum volume). The multivariate model was internally validated using bootstrapping (170 replications).

All analyses were performed using Stata V13 software (StataCorp LP, College Station, TX). P values were twotailed and considered significant when less than 0.05 .

\section{Results}

\section{Characterization of toxicity}

We observed acute G1, G2 and G3 GI toxicity in 96 patients (53.3\%), 19 patients (10.6\%) and 2 patients (1.1\%), respectively. No G4 or G5 GI toxicity was observed. Among these toxicities, $35.8 \%$ of patients had diarrhea ( $29.6 \% \mathrm{G} 1$ and $6.2 \% \mathrm{G} 2$ ) and $36.1 \%$ of patients had proctitis (32.2 \% G1, $3.3 \% \mathrm{G} 2$ and $0.6 \% \mathrm{G} 3)$.

\section{Predictors of acute Gl toxicity}

The median volumes of the rectum in \% and in cc receiving from $25 \mathrm{~Gy}$ to $75 \mathrm{~Gy}$ (increments of 5Gy) are presented in Table 2.

In the univariate analysis of the entire patient population, we found no relationship between any rectal volume parameters expressed in $\%$ and any acute GI toxicity $\geq$ grade1 ( $p$ from 0.12 to 0.92 ) (Table 2 ).

Conversely, when expressed in cc, all rectal volumes from V25 to V50 correlated significantly with acute GI toxicity $\mathrm{G} \geq 1$ (p from 0.018 to 0.045 ). Beyond 50Gy, no relationship was found between the volume of rectum expressed in cc and acute GI toxicity (from 55Gy to 75Gy, $p$-values ranged between 0.069 and 0.853 ) (Table 2).

The rAUC $_{25-50}$ calculated using the rectum volume expressed in cc correlated with any grade $\geq 1$ acute GI toxicity $(p=0.028)$ (Table 2$)$ while the $\mathrm{rAUC}_{25-50}$ calculated using rectum volume expressed in \% did not correlate with any acute GI toxicity (data not shown).

Multivariate logistic regression, which included the variables age, hormone therapy, TURP and rectum volume (cc), was used. Among these variables, only rectum volume expressed in cc correlated significantly with acute GI toxicity $(p=0.041)$. A Liu/Youden cutting method showed that patients with a $\mathrm{rAUC}_{25-50}>$ 794 cc.Gy were more likely to develop acute GI toxicity with IG-IMRT ( $p=0.020$, [95 \% CI: 1.16-5.46]) (Table 3). These results were validated by a bootstrapping method using 170 replications ( $p=0.019$; [95 \% CI: 1.16-5.42]).

\section{Discussion}

One of the major limits of DVSE is that the DVH curve can reach V70 by different paths, meaning that doses delivered before or beyond this specific endpoint might differ considerably for the same V70. Given this, for the same volume of rectum receiving a high dose, one patient may have a greater rectal volume irradiated at lower doses 
Table 2 Evaluation of relationship between DVSE and acute gastrointestinal toxicity using univariate logistic regression analysis

\begin{tabular}{|c|c|c|c|c|}
\hline Median [range] & $\begin{array}{l}\text { All patients } \\
n=180\end{array}$ & $\begin{array}{l}\text { Patients with acute toxicities } G^{b}=0 \\
n=63\end{array}$ & $\begin{array}{l}\text { Patients with acute toxicities } \mathrm{G} \geq 1 \\
n=117\end{array}$ & $p$-value \\
\hline \multicolumn{5}{|c|}{ Rectum volumes expressed in \% } \\
\hline $\mathrm{V} 25^{\mathrm{a}}$ & $68[27 ; 100]$ & $69.6[27 ; 100]$ & $67.7[31.6 ; 100]$ & 0.799 \\
\hline V30 & $60.9[24.2 ; 100]$ & $61.6[24.2 ; 100]$ & $60.7[25.5 ; 100]$ & 0.860 \\
\hline V35 & $51.9[21 ; 100]$ & $51.7[21.8 ; 99.6]$ & $52.1[21 ; 100]$ & 0.726 \\
\hline V40 & $44[17.6 ; 96.7]$ & $44.3[19.7 ; 85.3]$ & $43.9[17.6 ; 96.7]$ & 0.638 \\
\hline V45 & $35.2[13.1 ; 82.2]$ & $37.1[15.5 ; 62.8]$ & $34.8[13.1 ; 82.2]$ & 0.704 \\
\hline V50 & $27.8[8 ; 74.6]$ & $28.3[9.3 ; 52.9]$ & $27.7[8 ; 74.6]$ & 0.766 \\
\hline V55 & $22.4[5.4 ; 51.2]$ & $23[5.4 ; 43.6]$ & $22.3[6.1 ; 51.2]$ & 0.875 \\
\hline V60 & $17.2[2.7 ; 40.8]$ & $17.6[2.7 ; 35.1]$ & $17[3.4 ; 40.8]$ & 0.918 \\
\hline V65 & $11.8[1 ; 29.5]$ & $12.6[1 ; 29.5]$ & $11.5[1.2 ; 26.5]$ & 0.582 \\
\hline V70 & $7.3[0 ; 23.1]$ & $7.8[0 ; 23.1]$ & $6.7[0 ; 19.8]$ & 0.217 \\
\hline V75 & $0.9[0 ; 11.4]$ & $1.5[0 ; 11.4]$ & $0.7[0 ; 9.1]$ & 0.124 \\
\hline \multicolumn{5}{|c|}{ Rectum volumes expressed in cc } \\
\hline V25 & $53.5[20.1 ; 223]$ & $48.8[20.1 ; 114]$ & $57[20.6 ; 223]$ & 0.039 \\
\hline V30 & $47.5[19.5 ; 180.2]$ & $42.8[19.5 ; 99.8]$ & $50.9[19.8 ; 180.2]$ & 0.045 \\
\hline V35 & $41.7[16.7 ; 150]$ & $37.8[16.7 ; 86]$ & $42.6[17.4 ; 150]$ & 0.035 \\
\hline V40 & $36.1[11 ; 120.8]$ & $31.9[11 ; 79]$ & $37.4[13.9 ; 120.8]$ & 0.025 \\
\hline V45 & $29.8[8 ; 101.9]$ & $25.8[8 ; 69.1]$ & $31.6[10.2 ; 101.9]$ & 0.018 \\
\hline V50 & $24.1[5.9 ; 77]$ & $21.1[5.9 ; 58.1]$ & $25[7.6 ; 77]$ & 0.035 \\
\hline V55 & $19[4.4 ; 56.4]$ & $17.9[4.4 ; 47.8]$ & $19.7[5.8 ; 56.4]$ & 0.069 \\
\hline V60 & $14.6[2.4 ; 43.6]$ & $13.1[2.4 ; 36.3]$ & $15.2[3.1 ; 43.6]$ & 0.17 \\
\hline V65 & $10.2[0.9 ; 36.8]$ & $9.9[0.9 ; 28.7]$ & $11[1.3 ; 36.8]$ & 0.387 \\
\hline V70 & $5.7[0 ; 29.5]$ & $5.8[0 ; 20.2]$ & $5.7[0 ; 29.5]$ & 0.853 \\
\hline V75 & $0.8[0 ; 16.2]$ & $0.9[0 ; 8]$ & $0.6[0 ; 16.2]$ & 0.232 \\
\hline 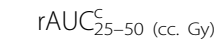 & $972.5[388.5 ; 3305.3]$ & 835.7 [394.3;2008.8] & $1002.4[388.5 ; 3305.3]$ & 0.028 \\
\hline
\end{tabular}

${ }^{\mathrm{a}} \mathrm{Vx}=$ volume of rectum receiving $\mathrm{xGy} ;{ }^{\mathrm{b}}$ Grade using CTC-AE V3.0 validated scale; ${ }^{\mathrm{C}}$ Area Under the Curve of rectal DHV between 25 and $50 \mathrm{~Gy}$

while another may have his rectum spared when evaluated according to the planning CT. For this reason, the QUANTEC recommended several DVSE in the context of dose escalation delivered using 3D conformational radiotherapy [4]. Recently, like us, Pederson et al. found a lack of any correlation between standard DVSE criteria and late GI or GU (genitourinary) toxicities, evaluated using RTOG and CTCAE V3.0 scales, induced by IMRT [13]. The authors suggested adapting rectum DVH, which correlated with late toxicities induced by IMRT. A new parameter related to acute toxicity has yet to be developed.

A number of preliminary clinical studies on high-dose IMRT and/or high-dose IGRT, like ours, reported very low rates of acute GI toxicity. In routine practice with IG-IMRT, acute toxicity is much more frequent than late toxicity and most patients have grade 1 or 2 toxicity only, suggesting that it would be better to figure out what dose/volume parameters best predict any acute GI toxicity ( $n=117$ in our series) rather than severe GI toxicity only. Although acute toxicities were prospectively recorded in the follow-up of all of the patients, one limitation of our study arises from the retrospective design, with drawbacks related to the post hoc scoring of toxicity using version 3 of the CTC scale.

Even though toxicity was prospectively scored, one drawback of our retrospective analyses concerns the major differences in scoring systems in the literature. Patientreported outcomes may be more clinically relevant and we therefore suggest conducting a new prospective study that includes both physician- and patient-reported outcomes.

Nevertheless we found a lower rate of acute G $\geq 2$ GI toxicity in our series (12\%), which was very similar to that observed by Kupelian et al. in 488 patients with daily IG-IMRT (11 \% of G2 acute rectal toxicity) [14] but lower than that observed by Wortel et al. in 260 patients treated with IMRT ( $29 \%$ of $G \geq 2$ acute rectal toxicity) [9]. These two studies used the RTOG toxicity scoring system. In another report, Singh et al. also confirmed less severe rectal symptoms with IGRT compared with non-IGRT [2]. 
Table 3 Optimal $r A \cup C_{25-50}$ Cut-off value related to acute Gl toxicity Grade $\geq 1$ determined using a ROC curve (multivariate logistic regression)

\begin{tabular}{|c|c|c|c|c|c|c|c|c|c|c|}
\hline & \multicolumn{4}{|l|}{ Univariate analysis } & \multicolumn{4}{|l|}{ Multivariate analysis } & \multicolumn{2}{|c|}{ Bootstrapping (170 rep) } \\
\hline & $\begin{array}{l}\text { Acute } \mathrm{Gl}^{\mathrm{a}} \text { toxicity } \\
\mathrm{G} \geq 1 / \mathrm{N}\end{array}$ & $\mathrm{OR}^{c}$ & $95 \% \mathrm{Cl}^{\mathrm{d}}$ & $\overline{p \text {-value }}$ & $\begin{array}{l}\text { Acute } G \text { toxicity } G \geq 1 / N \\
115 / 178\end{array}$ & OR & $95 \% \mathrm{Cl}$ & $\overline{p \text {-value }}$ & $95 \% \mathrm{Cl}$ & $p$-value \\
\hline \multicolumn{11}{|l|}{ rAUC $C_{25-50}^{\mathrm{b}}$ rectum } \\
\hline \multicolumn{11}{|l|}{ Liu/Youden cutting methods } \\
\hline$<=794$ cc.Gy & $29 / 59$ & 1 & & 0.002 & $28 / 58$ & 1 & & 0.020 & & 0.019 \\
\hline > 794 cc.Gy & $88 / 121$ & 2.76 & {$[1.44 ; 5.28]$} & & $87 / 120$ & 2.51 & {$[1.16 ; 5.46]$} & & {$[1.16 ; 5.42]$} & \\
\hline \multicolumn{11}{|l|}{ Adjustment variables } \\
\hline \multicolumn{11}{|l|}{ Age } \\
\hline$<70$ years & $57 / 82$ & 1 & & 0.247 & $57 / 82$ & 1 & & 0.233 & & 0.226 \\
\hline$>=70$ years & $60 / 98$ & 0.69 & {$[0.37 ; 1.29]$} & & $58 / 96$ & 0.67 & {$[0.35 ; 1.29]$} & & {$[0.35 ; 1.28]$} & \\
\hline \multicolumn{11}{|l|}{ TURPe } \\
\hline No & $95 / 143$ & 1 & & 0.305 & $95 / 143$ & 1 & & 0.423 & & 0.459 \\
\hline Yes & $20 / 35$ & 0.67 & {$[0.32 ; 1.43]$} & & $20 / 35$ & 0.72 & {$[0.33 ; 1.59]$} & & {$[0.31 ; 1.7]$} & \\
\hline \multicolumn{11}{|l|}{$H T^{f}$} \\
\hline No & $65 / 104$ & 1 & & 0.411 & $64 / 103$ & 1 & & 0.757 & & 0.755 \\
\hline Yes & $52 / 76$ & 1.3 & {$[0.7 ; 2.43]$} & & $51 / 75$ & 1.11 & {$[0.57 ; 2.16]$} & & {$[0.57 ; 2.15]$} & \\
\hline \multirow[t]{2}{*}{ Rectum Volume (cc) per unit } & & 1 & & 0.041 & & 1 & & 0.558 & & 0.570 \\
\hline & & 1.01 & {$[1 ; 1.02]$} & & & 1 & {$[0.99 ; 1.01]$} & & {$[0.99 ; 1.01]$} & \\
\hline
\end{tabular}

agastrointestinal; ${ }^{b}$ Area Under the Curve of rectal DHV between 25 and 50Gy, ${ }^{\text {oddds ratio; }}{ }^{\mathrm{d}} 95 \%$ confidence interval; ${ }^{\mathrm{e}}$ transurethral resection of the prostate; ${ }^{\mathrm{f}}$ hormonotherapy 
We believe that daily IG-IMRT with a sharper dose gradient, thanks to daily repositioning on the prostate isocenter, may ensure that high doses are only delivered to the same small areas of the rectum as is the case with brachytherapy. Our results are in keeping with this hypothesis as we found that only the absolute volume of the rectum (but not the relative volume of the rectum) between 25Gy and 50Gy correlated with acute GI toxicity when patients were treated with daily on-line IG-IMRT.

Moreover, these results suggest that intermediate doses delivered to the rectum may be more relevant than high doses with daily IG-IMRT.

\section{Conclusions}

We have used a simple method to identify a new single parameter derived from the $\mathrm{DVH}$, in contrast to several DVSE, that predicts acute GI toxicity: the $\mathrm{rAUC}_{25-50}$ is a user-friendly tool that can be implemented in any radiation oncology department worldwide.

We recommend that the $\mathrm{rAUC}_{25-50}$ of the entire rectum should not exceed 794 cc.Gy. This new predictive parameter for acute GI toxicity should be validated through a prospective study.

\section{Abbrevations}

3D-CRT: 3-dimensional conformal radiation therapy; CTCAE: Common terminology criteria for adverse events; CTV: Clinical target volume; DVH: Dose volume histogram; DVSE: Dose/volume specific endpoints; Gl: Gastrointestinal; GTV: Gross tumor volume; GU: Genito-urinary; HT: Hormonotherapy; IG-IMRT: Image-guidance intensity modulated radiation therapy; OAR: Organs at risk; PCa: Prostate cancer; PTV: Planning target volume; rAUC: Area under the rectal DVH curve; rAUC $25-50$ : rAUC for doses ranging between 25Gy and 50Gy; RTOG: Radiation Therapy Oncology Group; TURP: Transurethral resection of the prostate; $\checkmark x$ : Volume of rectum receiving $x G y$

\section{Acknowledgements}

We want to thank Philip Bastable for his review and corrections of the wording in this manuscript.

\section{Funding}

None.

\section{Availability of data and materials}

The data will not be shared as other original publications are under consideration

\section{Authors' contributions}

CM, PW and GC designed the study. CM, PW, MG, CD, SN, FM and EM contributed to data acquisition and analysis. CM and GC drafted the manuscript and PM, PW, EM and MG revised the manuscript critically. All authors have approved the final version to be published.

\section{Competing interests}

The authors declare that they have no competing interests.

\section{Consent for publication}

Not applicable.

\section{Ethics approval and consent to participate}

The institutional committee of the Centre Georges François Leclerc on human research approved the study. All of the patients analyzed in the present study had provided written consent in our establishment for the use of their clinical data to conduct retrospective studies.

\section{Author details}

1Department of Radiation Oncology, Centre Georges Francois Leclerc, 1, rue du Pr Marion, 21049 Dijon, France. ${ }^{2}$ Medical Imaging Group, Laboratory of Electronics, Computer Science and Imaging, (Le2I), CNRS 6306, University of Burgundy, 21000 Dijon, France. ${ }^{3}$ Department of Biostatistics, Centre Georges Francois Leclerc, 21049 Dijon, France.

Received: 10 February 2016 Accepted: 27 October 2016

Published online: 04 November 2016

\section{References}

1. Kok D, Gill S, Bressel M, Byrne K, Kron T, Fox C, et al. Late toxicity and biochemical control in 554 prostate cancer patients treated with and without dose escalated image guided radiotherapy. Radiother Oncol. 2013;107(2):140-6.

2. Singh J, Greer PB, White MA, Parker J, Patterson J, Tang Cl, et al. Treatmentrelated morbidity in prostate cancer: a comparison of 3-dimensional conformal radiation therapy with and without image guidance using implanted fiducial markers. Int J Radiat Oncol Biol Phys. 2013;85(4):1018-23.

3. Zelefsky MJ, Kollmeier M, Cox B, Fidaleo A, Sperling D, Pei X, et al. Improved clinical outcomes with high-dose image guided radiotherapy compared with non-IGRT for the treatment of clinically localized prostate cancer. Int J Radiat Oncol Biol Phys. 2012;84(1):125-9.

4. Michalski JM, Gay H, Jackson A, Tucker SL, Deasy JO. Radiation dose-volume effects in radiation-induced rectal injury. Int J Radiat Oncol Biol Phys. 2010; 76(3 Suppl):S123-9. Pubmed Central PMCID: 3319467.

5. Boersma LJ, van den Brink M, Bruce AM, Shouman T, Gras L, te Velde A, et al. Estimation of the incidence of late bladder and rectum complications after high-dose (70-78 GY) conformal radiotherapy for prostate cancer, using dosevolume histograms. Int J Radiat Oncol Biol Phys. 1998;41(1):83-92.

6. Chan LW, Xia P, Gottschalk AR, Akazawa M, Scala M, Pickett B, et al. Proposed rectal dose constraints for patients undergoing definitive whole pelvic radiotherapy for clinically localized prostate cancer. Int J Radiat Oncol Biol Phys. 2008;72(1):69-77.

7. Fiorino C, Valdagni R, Rancati T, Sanguineti G. Dose-volume effects for normal tissues in external radiotherapy: pelvis. Radiother Oncol. 2009;93(2):153-67.

8. Huang EH, Pollack A, Levy L, Starkschall G, Dong L, Rosen I, et al. Late rectal toxicity: dose-volume effects of conformal radiotherapy for prostate cancer. Int J Radiat Oncol Biol Phys. 2002;54(5):1314-21.

9. Wortel RC, Incrocci L, Pos FJ, Lebesque JV, Witte MG, van der Heide UA, et al. Acute toxicity after image-guided intensity modulated radiation therapy compared to 3D conformal radiation therapy in prostate cancer patients. Int J Radiat Oncol Biol Phys. 2015;91(4):737-44.

10. Alongi F, Fiorino C, Cozzarini C, Broggi S, Perna L, Cattaneo GM, et al. IMRT significantly reduces acute toxicity of whole-pelvis irradiation in patients treated with post-operative adjuvant or salvage radiotherapy after radical prostatectomy. Radiother Oncol. 2009;93(2):207-12.

11. Crehange G, Mirjolet C, Gauthier M, Martin E, Truc G, Peignaux-Casasnovas $\mathrm{K}$, et al. Clinical impact of margin reduction on late toxicity and short-term biochemical control for patients treated with daily on-line image guided IMRT for prostate cancer. Radiother Oncol. 2012;103(2):244-6.

12. Peignaux K, Truc G, Barillot I, Ammor A, Naudy S, Crehange G, et al. Clinical assessment of the use of the Sonarray system for daily prostate localization. Radiother Oncol. 2006;81(2):176-8.

13. Pederson AW, Fricano J, Correa D, Pelizzari CA, Liauw SL. Late toxicity after intensity-modulated radiation therapy for localized prostate cancer: an exploration of dose-volume histogram parameters to limit genitourinary and gastrointestinal toxicity. Int J Radiat Oncol Biol Phys. 2012;82(1):235-41.

14. Kupelian PA, Willoughby TR, Reddy CA, Klein EA, Mahadevan A. Impact of image guidance on outcomes after external beam radiotherapy for localized prostate cancer. Int J Radiat Oncol Biol Phys. 2008;70(4):1146-50. 